\title{
Electronic Structure of Metallocene-based Polymers
}

\author{
By Yukihito MATSUURA, ${ }^{*}$ Ryota ODANI, and Yuki TANAKA
}

\begin{abstract}
The band structures of metallocene-based polymers in which the nearest metalloecenes were directly linked by a C-C bond have been determined by employing the tight-binding crystal orbital (CO) method. In particular, we determined the band structures of metallocene-based alternate copolymers whose metal sequences in a unit cell were $\mathrm{Fe}-\mathrm{Co}, \mathrm{Fe}-\mathrm{Fe}-\mathrm{Co}-\mathrm{Co}$, $\mathrm{Fe}-\mathrm{Ni}$, $\mathrm{Ru}-\mathrm{Co}$ and Os-Co. The band structures of alternate copolymers of Fe-Co and Fe-Ni possessed a partially occupied (PO) band whose crystal orbitals were extremely localized at the $3 \mathrm{~d}$ orbitals of cobalt or nickel atom. Alternate copolymers of Ru-Co and Os-Co had the same tendency as that of band structure of Fe-Co. Therefore, it was clear that the band structures of the metallocene-based alternate copolymers were considered to be a superposition of the polymetallocenylenes. Furthermore, we examined the effect of inserting the bridging part of boron-, phosphorus-, and sulfur bridges between the nearest ferrocenes upon the band structures of ferrocene-based polymers. The electronic structure of the boron-bridged polymer was similar to that of the silicon-bridged polymer whose band structure was determined in our previous study. On the other hand, the phosphorus- and sulfur-bridged polymers yielded two degenerated HO bands whose COs were localized at P or S $2 \mathrm{p}$ orbitals. KEY WORDS: Metallocene / Polymer / Band Structure /
\end{abstract}

Metallocene-based oligomers and polymers have attracted considerable attention for more than a decade. ${ }^{1}$ In particular, ferrocene-based polymers have been synthesized using various synthetic methods. ${ }^{2}$ Polyferrocenylene ( $\left.\mathrm{PFeC}\right)$, whose nearest ferrocenes are directly linked to each other by the $\mathrm{C}-\mathrm{C}$ bond, has been synthesized, ${ }^{3}$ and its electronic structure has been found to have the characteristic of a semiconductor by employing tight-binding band calculations., ${ }^{4,5}$ Our band calculations have shown that the semiconductor characteristic was due to the partially delocalized $\pi$ electrons in the fulvalene units. ${ }^{5}$ It has been reported that ferrocene-based polymers with unsaturated carbon bridges exhibit a strong interaction between the nearest unit cells. ${ }^{1}$ Furthermore, we have designed metallocene-based polymers such as polycobaltocenylene (PCoC) and polynickelocenylene $(\mathrm{PNiC})$ in which the nearest metallocenes linked by the $\mathrm{C}-\mathrm{C}$ bond. Further we have examined their electronic structures by employing the tight-binding band calculations. ${ }^{6}$

Recently, several heterobimetallic bimetallocenes have been obtained. ${ }^{7}$ The replacement of $\mathrm{Fe}$ with $\mathrm{Co}$ or Ni yields an odd number of electrons, which are expected to result in strange magnetic properties. The orbital interactions in $\mathrm{Fe} / \mathrm{Co}$ heterobimetallocenes were analyzed by using NMR, Mössbauer and magnetic characterization, and it was found that some compounds exhibited a strong magnetic interaction between $\mathrm{Fe}$ and $\mathrm{Co}$ or Ni. Termetallocenes with the metal sequences such as Ni-Fe-Ni, Fe-Co-Fe, Co-Fe-Co, Co-Ru-Co, Co-Os-Co and $\mathrm{Rh}-\mathrm{Fe}-\mathrm{Co}$ have also been synthesized, and their crystal structures and electrochemical properties have been studied in details. ${ }^{8}$ Since metallocenes other than ferrocene possess some unpaired electrons that can result in strange magnetic properties, we are naturally interested in its synthesis for linking infinite heteronuclear structures of polymetallocenylene.
On the other hand, ferrocene-based polymers with heteroatom bridges ( $\mathrm{Si}, \mathrm{Ge}, \mathrm{Sn}, \mathrm{P}, \mathrm{S}$ etc.) have been synthesized by the thermal ring-opening polymerization of strained cyclic, heteroatom-bridged ${ }^{1}$ ferrocenophanes. ${ }^{2}$ These polymers were found to exhibit a strong interaction between the nearest unit cells, which was confirmed by electrochemical analyses. Furthermore, we have found that the strong correlation between the nearest ferrocenes is strongly dependent on the density of states (DOS) of ferrocene-based polymers with silicon, germanium or stannum bridges. ${ }^{9}$ The electrical and magnetic properties of ferrocene-based polymers with heteroatombridges other than polyferrocenylene (PFS) have not yet been studied in details. However, their strong interaction may lead to some interesting properties applicable to electronic or magnetic devices.

As mentioned above, either the replacement of transition metals or insertion of bridging parts can be expected to provide an electronic or magnetic modification for the ferrocene-based polymers due to the strong interaction between the nearest metallocenes. Therefore, in this study, we have modified $\mathrm{PFeC}$ in the following two ways: (1) replacement of iron atoms in $\mathrm{PFeC}$ with other transition metals and (2) insertion of heteroatom bridges such as boron, phosphorus and sulfur between the nearest $\mathrm{Cp}$ rings. We have also examined their band structures by employing tight-binding band calculations.

\section{CALCULATION METHOD}

The one-dimensional tight-binding crystal orbital calculations were performed using the YAeHMOP program. ${ }^{10}$ The atomic parameters $\left(H_{\mathrm{ii}}=\right.$ orbital energy, $\zeta=$ Slater exponent $)$ for the approximation are provided in Table I. It has been reported that a so-called counterintuitive orbital mixing is 
Table I. Parameters for $\mathrm{H}, \mathrm{B}, \mathrm{C}, \mathrm{Si}, \mathrm{P}, \mathrm{S}, \mathrm{Fe}, \mathrm{Co}, \mathrm{Ni}, \mathrm{Ru}$, and Os atoms

\begin{tabular}{|c|c|c|c|c|c|c|}
\hline atom & orbital & $H_{\mathrm{ii}}(\mathrm{eV})$ & $\zeta_{1}$ & $\zeta_{2}$ & $C_{1}{ }^{a}$ & $C_{2}{ }^{a}$ \\
\hline $\mathrm{H}$ & $1 \mathrm{~s}$ & -13.60 & 1.3000 & & & \\
\hline \multirow[t]{2}{*}{$B$} & $2 s$ & -15.20 & 1.3000 & & & \\
\hline & $2 p$ & -8.50 & 1.3000 & & & \\
\hline \multirow[t]{2}{*}{$C$} & $2 s$ & -21.40 & 1.6250 & & & \\
\hline & $2 p$ & -11.40 & 1.6250 & & & \\
\hline \multirow[t]{2}{*}{$\mathrm{Si}$} & $3 s$ & -17.30 & 1.3830 & & & \\
\hline & $3 p$ & -9.20 & 1.3830 & & & \\
\hline \multirow[t]{2}{*}{$P$} & $3 s$ & -18.60 & 1.7500 & & & \\
\hline & $3 p$ & -14.00 & 1.3000 & & & \\
\hline \multirow[t]{2}{*}{$S$} & $3 s$ & -20.00 & 2.1200 & & & \\
\hline & $3 p$ & -11.00 & 1.8270 & & & \\
\hline \multirow[t]{3}{*}{$\mathrm{Fe}$} & $4 \mathrm{~s}$ & -9.10 & 1.9000 & & & \\
\hline & $4 p$ & -5.32 & 1.9000 & & & \\
\hline & $3 d$ & -12.60 & 5.3500 & 2.0000 & 0.5505 & 0.6260 \\
\hline \multirow[t]{3}{*}{ Co } & $4 \mathrm{~s}$ & -9.21 & 2.0000 & & & \\
\hline & $4 p$ & -5.29 & 2.0000 & & & \\
\hline & $3 d$ & -13.18 & 5.5500 & 2.1000 & 0.5679 & 0.6059 \\
\hline \multirow[t]{3}{*}{$\mathrm{Ni}$} & $4 \mathrm{~s}$ & -10.95 & 2.1000 & & & \\
\hline & $4 p$ & -6.27 & 2.1000 & & & \\
\hline & $3 d$ & -14.20 & 5.7500 & 2.3000 & 0.5493 & 0.6082 \\
\hline \multirow[t]{3}{*}{$\mathrm{Ru}$} & $5 s$ & -10.40 & 2.0800 & & & \\
\hline & $5 p$ & -6.80 & 2.0400 & & & \\
\hline & $4 d$ & -14.90 & 5.3800 & 2.3000 & 0.5342 & 0.6368 \\
\hline \multirow[t]{3}{*}{ Os } & $6 s$ & -8.17 & 2.4520 & & & \\
\hline & $6 p$ & -4.81 & 2.4290 & & & \\
\hline & $5 d$ & -11.84 & 5.5710 & 2.4160 & 0.6372 & 0.5598 \\
\hline
\end{tabular}

${ }^{\text {a }}$ Coefficients used in the doble- $\zeta$ expansion of the $d$ orbitals.

induced when using the approximation especially in calculations of transition metal complexes. ${ }^{11}$ Therefore, a modified Wolfsberg-Helmholz formula was employed for the off diagonal matrix elements $H_{\mathrm{ij}}$ as follows:

$$
\begin{aligned}
& H_{\mathrm{ij}}=1 / 2 K S_{\mathrm{ij}}\left(H_{\mathrm{ii}}+H_{\mathrm{jj}}\right) \\
& K=k+\Delta^{2}+\Delta^{4}(1-k) ; k=1.75 \\
& \Delta=\left(H_{\mathrm{ii}}-H_{\mathrm{jj}}\right) /\left(H_{\mathrm{ii}}+H_{\mathrm{jj}}\right)
\end{aligned}
$$

with the usual notation. For calculating the band structures, twenty representative wave vectors $(k)$ were chosen from 0 to $\pi / a$ ( $a$ is the unit cell length) with regular intervals, and neighbouring unit cells as far as the fifth nearest cells were considered.

The polymers chosen in this report were directly linked metallocene-based polymers as shown in Figure 1. At first, we have designed PFeC (Figure 1(a)), an alternate copolymer of $\mathrm{PFeC}$ and $\mathrm{PCoC}$ (PFeCoC) (Figure 1(b)), an alternate copolymer of $\mathrm{PFeC}$ and $\mathrm{PCoC}$ whose unit cell consisted of two ferrocenylene and two cobaltocenylene $\left(\mathrm{PFe}_{2} \mathrm{Co}_{2} \mathrm{C}\right)$ (Figure 1(c)) and an alternate copolymer of $\mathrm{PFeC}$ and $\mathrm{PNiC}$ (PFeNiC) (Figure 1(d)), and have calculated their band structures. Subsequently, we tried to replace Fe with $\mathrm{Ru}$ or Os in polymetallocenylene to obtain the model of polyruthenocenylene (PRuC) (Figure 1(e)), an alternate copolymer of $\mathrm{PRuC}$ and $\mathrm{PCoC}$ (PRuCoC) (Figure 1(f)), polyosmocenylene (POsC) (Figure $1(\mathrm{~g})$ ) and an alternate copolymer of POsC and PCoC (POsCoC) (Figure 1(h)). Furthermore, we have also designed ferrocene-based polymer with heteroatom bridges
(PFB), ferrocene-based polymer with phosphorus bridges $(\mathrm{PFPh})$, and ferrocene-based polymer with sulfur bridges $(\mathrm{PFSu})$, as described in Figure 1(i), (j), and (k).

In this study, the chemical structures of the metallocenebased polymers were determined using typical chemical structures of the organic compounds described in references. $^{12,13}$ When we constructed a structural model of the unit cell of the polymers, the symmetry of the unit cell of metallocene was determined to be $C_{2 h}$ and the angle of the $\mathrm{C}-\mathrm{H}$ bond outside the $\mathrm{Cp}$ ring was $0^{\circ}$. Furthermore, when we determined the distance between the transition metal atom and the $\mathrm{Cp}$ ring, we referred to the results calculated at the B3LYP DZP level. ${ }^{14}$ The chemical structures of the metallocene moieties are described in our previous papers. ${ }^{6,9}$ The bond lengths of the heteroatom bridged polymers were determined as following: $\mathrm{C}-\mathrm{B}=1.43 \AA$; $\mathrm{C}-\mathrm{P}=1.84 \AA$; $\mathrm{C}-\mathrm{S}=1.92 \AA$.

\section{RESULTS AND DISCUSSION}

\section{Replacement of Transition Metals}

Figure 2(a) shows the band structure and density of states (DOS) of PFeC. Several bands near the Fermi level are degenerated at $\mathrm{k}=\pi / a$ due to the folding relationship ${ }^{15}$ of a unit cell of PFeC. ${ }^{9}$ Its band structure exhibits the characteristics of a semiconductor character, and its COs near the Fermi level are assigned as the same as that in reference. ${ }^{9}$ When one iron atom in a unit cell of $\mathrm{PFeC}$ is replaced with one cobalt atom, we can design $\mathrm{PFeCoC}$, as described in Figure 1(b). Figure 2(b) shows the band structure of PFeCoC. Some energy levels degenerated at $\mathrm{k}=\pi / a$ of $\mathrm{PFeC}$ were split into two energy levels due to the lowering symmetry of the unit cells in $\mathrm{PFeCoC}$. The highest occupied (HO) band of PFeCoC mainly consisted of $\pi$ orbitals of the $\mathrm{Cp}$ ring. The PO and the lowest unoccupied (LU) bands are mainly formed by Co $3 \mathrm{~d}_{\mathrm{yz}}$ orbitals. Projected DOS (PDOS) for Co atoms in Figure 2(b) also indicates that the PO and LU bands are formed by atomic orbitals of Co. Since the COs near the Fermi level are shown in our previous papers, ${ }^{5,6}$ PDOS of Co $3 \mathrm{~d}$ orbitals are not shown in this paper. On the other hand, as described in PDOS for $\mathrm{Fe}$ atoms (Figure 2(b)), the energy levels of the LU+1 and LU+2 bands, whose COs are formed by Fe $3 d_{y z}$ and $3 d_{x z}$ orbitals, do not vary with the change in the transition metal atoms. As a result, replacement of $\mathrm{Fe}$ with $\mathrm{Co}$ in $\mathrm{PFeC}$ results in a localized partially occupied (PO) band in a band structure of PFeCoC. For reference, we have also calculated the band structure of $\mathrm{PFe}_{2} \mathrm{Co}_{2} \mathrm{C}$, as shown in Figure $1(\mathrm{c}) . \mathrm{PFe}_{2} \mathrm{Co}_{2} \mathrm{C}$ exhibits the characteristics of a semiconductor character due to the even number of the electrons in the unit cell. The HOCO and LUCO of $\mathrm{PFe}_{2} \mathrm{Co}_{2} \mathrm{C}$ consisted of $\mathrm{Co} 3 \mathrm{~d}_{\mathrm{xz}}$ orbitals and the next $\mathrm{HOCO}$ is formed by $\pi$ orbitals of the $\mathrm{Cp}$ rings. Therefore, $\mathrm{PFe}_{2} \mathrm{Co}_{2} \mathrm{C}$ is likely to possess similar electrical properties as $\mathrm{PFeCoC}$.

When nickel atoms are introduced into a polymer structure of PFeC, we can obtain PFeNiC, as shown in Figure 1(d). The energy levels of the $\mathrm{PO}$ and $\mathrm{LU}$ bands of PFeNiC decrease significantly because the energy levels of the $\mathrm{Ni} 3 \mathrm{~d}$ orbitals are deeper than those of $\mathrm{PFeC}$ and $\mathrm{PFeCoC}$. The PDOS for $\mathrm{Ni}$ 
(a)

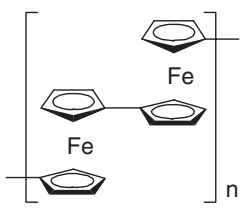

(d)

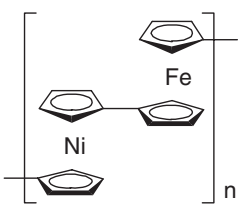

(g)

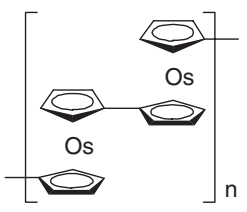

(b)

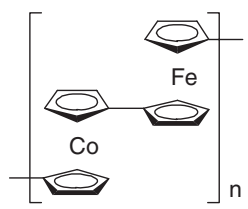

(e)
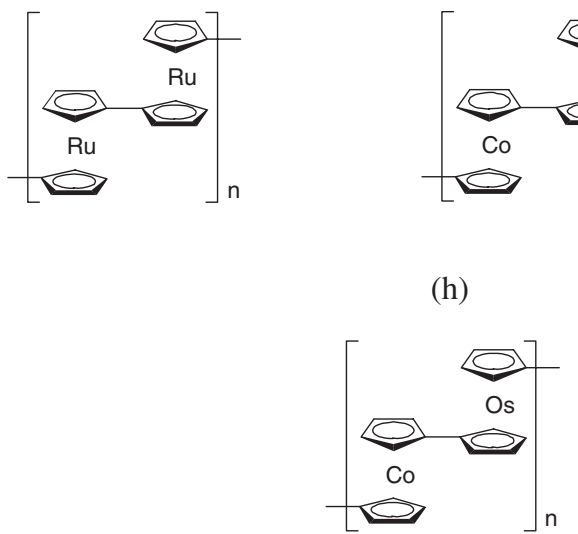

(c)

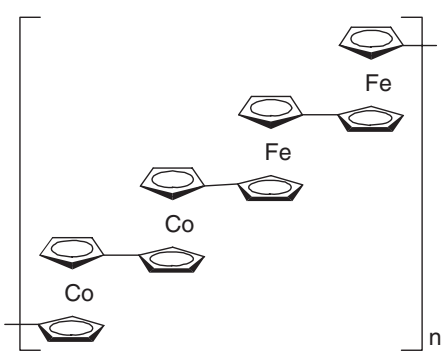

(f)

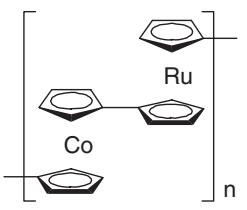

(h) (i)

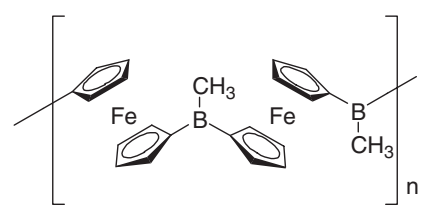

(j)

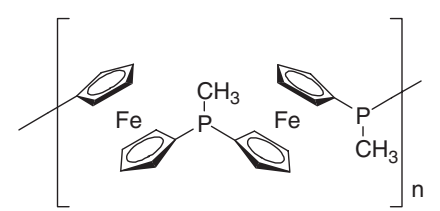

(k)

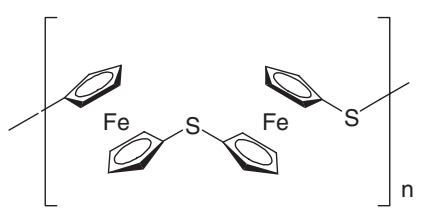

Figure 1. Chemical structures of (a) PFeC, (b) PFeCoC, (c) $\mathrm{PFe}_{2} \mathrm{Co}_{2} \mathrm{C}$, (d) PFeNiC, (e) PRuC, (f) PRuCoC, (g) POsC, (h) POsCoC, (i) PFB, (j) PFPh, and (h) PFSu.

atoms of $\mathrm{PFeNiC}$ also indicates that the $\mathrm{HO}$ and $\mathrm{PO}$ bands consisted of atomic orbitals of $\mathrm{Ni}$. The $\mathrm{PO}$ bandwidth is narrow, and hence the electrons at this band are localized at $\mathrm{Ni}$ atoms. On the other hand, the energy levels of the HO-1 band do not change. This is because the COs of the HO- 1 band of $\mathrm{PFeNiC}$ are mainly formed by the $\pi$ orbitals of the Cp rings, whose energy levels could not be affected by varying the transition atoms.

Figure 3(a) shows a band structure of PRuC. As described in our previous paper, ${ }^{6}$ the energy levels of the $\mathrm{HO}$ band of PRuC decrease and those of the LU band increase in comparison to those of $\mathrm{PFeC}$ to increase the band gap of PRuC. The CO patterns at the bands near the Fermi level of PRuC are similar to those of PFeC, the HOCO are formed by the $\pi$ orbitals of the $\mathrm{Cp}$ ring and LUCO $\mathrm{Ru} 4 \mathrm{~d}_{\mathrm{yz}}$ with a small contribution of $\pi$ orbitals of the $\mathrm{Cp}$ ring. The total DOS and the PDOS for $\mathrm{Ru}$ atoms of PRuC also confirm the character of its $\mathrm{HO}$ and $\mathrm{LU}$ bands. If one of the $\mathrm{Ru}$ atoms in a unit cell of $\mathrm{PRuC}$ is replaced with a Co atom, we can design an alternate copolymer of PRuCoC. Figure 3(b) shows a band structure of PRuCoC. There is a PO band whose COs are formed by Co $3 \mathrm{~d}$ orbitals, and its bandwidth is very small. The presence of electrons at the PO band results in the localized states in PRuCoC. Although the charge mobilities of PRuCoC are very low, its band gap decreases in order to increase the electrical conductivity of PRuCoC.

Figure 3(c) shows a band structure of POsC. The HOCO consists of the Os $5 \mathrm{~d}_{\mathrm{z}}{ }^{2}$ orbital, while the LUCO consists of the Os $5 \mathrm{~d}_{\mathrm{yz}}$ orbital. The $\pi$ orbitals of the $\mathrm{Cp}$ rings are located at a deep energy level of around $-12 \mathrm{eV}$. When compared with the band structures of PFeC and PRuC, the energy bands of POsC are very flat. This is due to the localization of Os $5 \mathrm{~d}$ electrons. Figure 3(d) shows the band structure of POsCoC. POsCoC also possessed PO band whose COs are localized at Co. Although POsCoC possesses a small band gap, its charge mobilities are likely to be very low. 


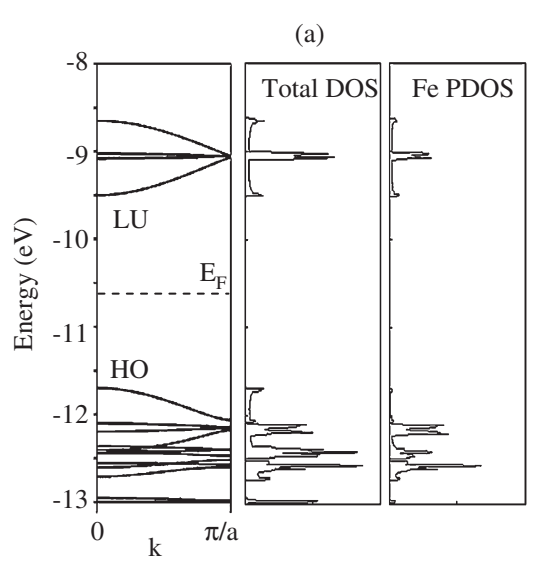

(c)

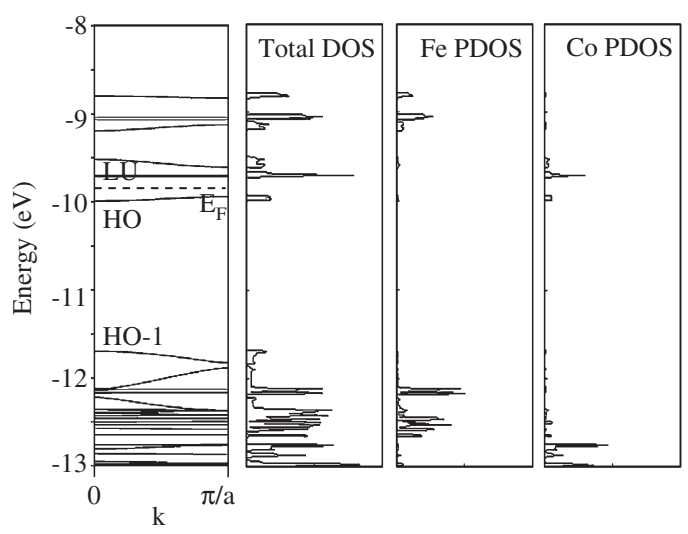

(b)

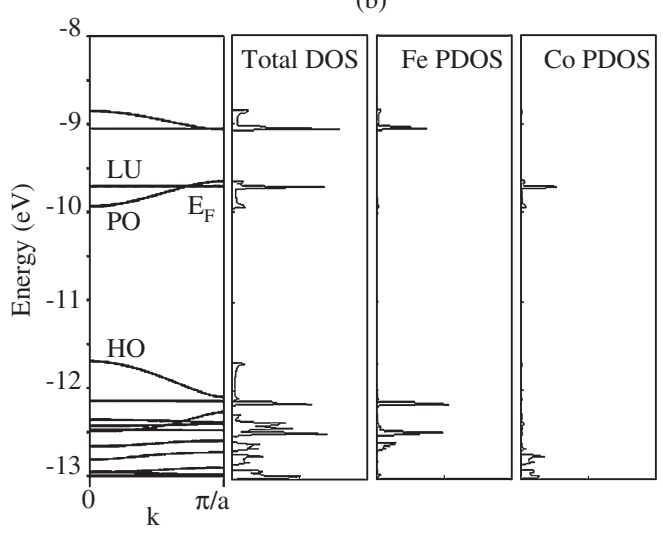

(d)

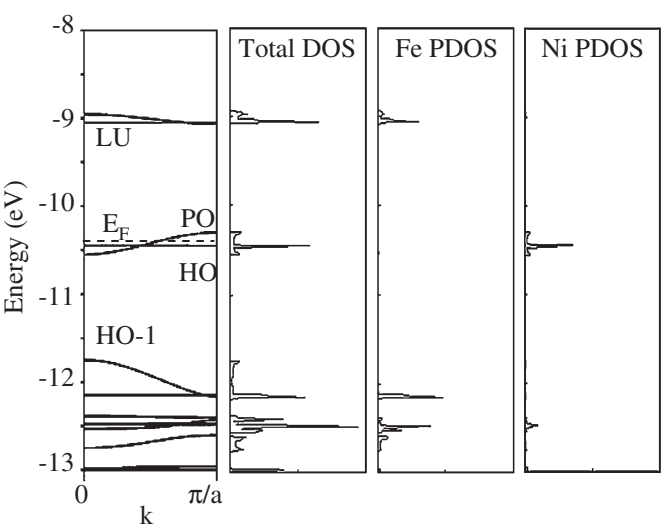

Figure 2. Band structures, total DOS and PDOS of (a) PFeC, (b) PFeCoC, (c) $\mathrm{PFe}_{2} \mathrm{Co}_{2} \mathrm{C}$ and (d) PFeNiC.

As a result, the band structures of the alternate copolymers consist of two superpositioned metallocene-based polymers as same as that of the molecular orbitals of the ferrocenyl cobaltocenium dimmer in reference. ${ }^{16}$

\section{Replacing the Bridging Parts}

Subsequently, we have examined ferrocene-based polymers that have bridging parts between the nearest ferrocenes. As described in reference, ${ }^{9}$ we have already calculated the band structure of PFS having silicon bridges. Its band structure exhibits the characteristics of a semiconductor character whose $\mathrm{CO}$ near the Fermi level consists of the Fe $3 \mathrm{~d}$ orbital with a small contribution from the $\pi$ orbital of the $\mathrm{Cp}$ ring. Moreover, its $\mathrm{HO}$ and LU bandwidths are very narrow. We replace the silicon-bridges in PFS with boron-bridges, as shown in Figure 1(i). The HO bandwidth of PFB is very narrow, and its HOCO mainly consists of $\mathrm{Fe} 3 \mathrm{~d}_{\mathrm{z}}{ }^{2}$ orbitals. The LUCO contains $\mathrm{Fe} 3 \mathrm{~d}_{\mathrm{yz}}$ orbitals with a small contribution from the $\pi$ orbitals of the $\mathrm{Cp}$ rings. Furthermore, the band gap of PFB is almost the same as that of PFS. Therefore, replacing the silicon bridge with the boron bridge is not efficient to improve the electronic properties of PFS.

When we replace the silicon bridges of PFS with phosphorus bridges, PFPh yields two filled $\mathrm{HO}$ bands of around $-11.5 \mathrm{eV}$, which are almost degenerated. The $\mathrm{HO}$ bands consists of $\mathrm{P} 2 \mathrm{p}$ orbitals. It is reported that $\mathrm{PFPh}$ is so reactive that it results in cross-linking. ${ }^{2}$ From the analyses of the $\mathrm{CO}$ of $\mathrm{PFPh}$, the reason for the cross-linking is due to the localized $\mathrm{P} 2 \mathrm{p}$ orbitals of PFPh. The character of the LU band is the same as that of PFS. From the band calculation, it is clear that replacing silicon bridges with phosphorus bridges is efficient to decrease the band gap.

As shown in Figure 4(c), a band structure of PFSu also has two degenerated $\mathrm{HO}$ bands of around $-10.5 \mathrm{eV}$. The energy level of PFSu is much higher than that of PFPh, and hence, the cross-linking or oxidation of the sulfur atoms may easily occur as compared with the other ferrocene-based polymers. The next HOCO consists of $\mathrm{p} \sigma$ bonding between the sulfur atom and the $\alpha$-carbon of the $\mathrm{Cp}$ ring, and hence the HO- 1 band of $\mathrm{PFBu}$ is stabilized to locate a deeper energy level than that of its HOCO. As in the case of PFS, these ferrocene-based polymers possess a narrow width of the $\mathrm{HO}$ and LU band, and hence the electrical conduction of these ferrocene-based polymers can also explain the small polaron hopping mechanism. ${ }^{17}$

\section{CONCLUSION}

We have examined the electronic structures of various metallocene-based polymers. Replacing the iron atom of a unit cell of $\mathrm{PFeC}$ with $\mathrm{Co}$ or $\mathrm{Ni}$ causes a split in the degenerated 
(a)

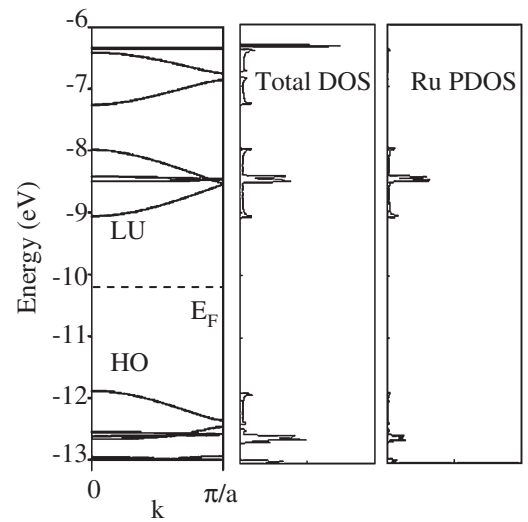

(c)

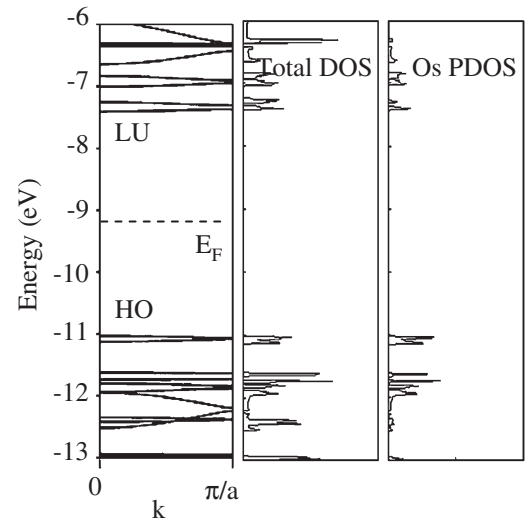

(b)

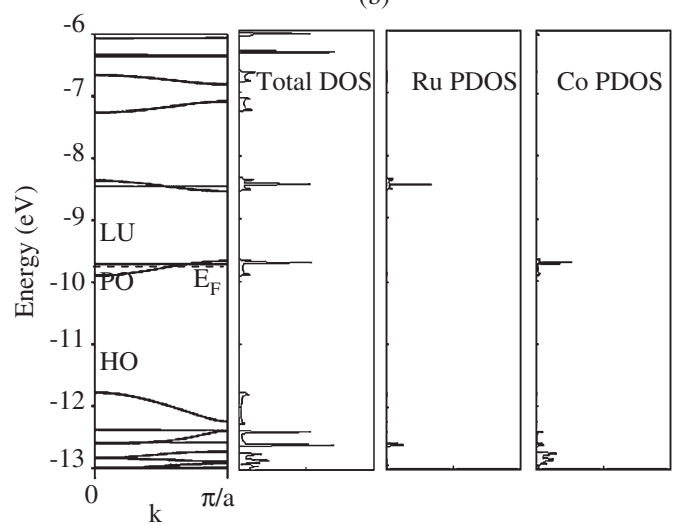

(d)

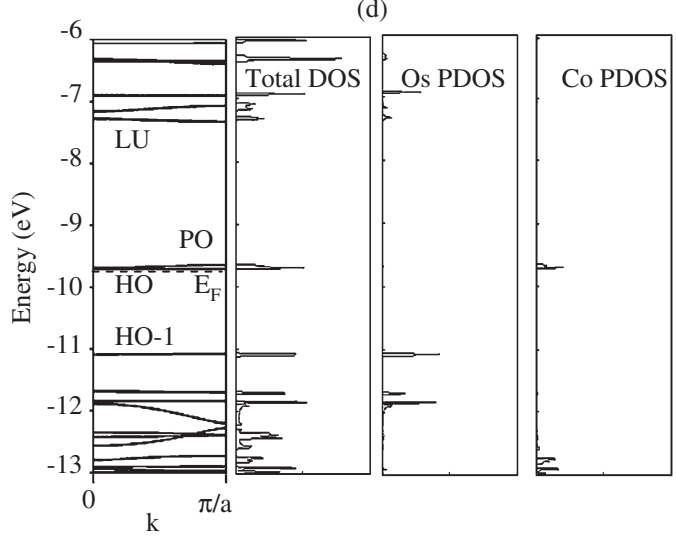

Figure 3. Band structures, total DOS and PDOS of (a) PRuC, (b) PRuCoC, (c) POsC and (d) POsCoC.

(a)

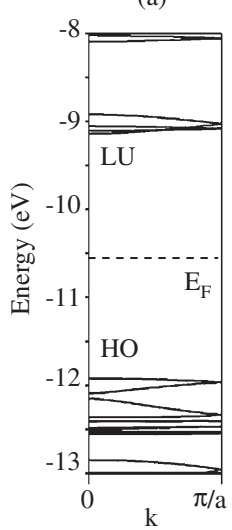

(b)

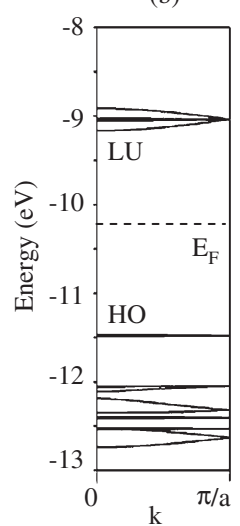

(c)

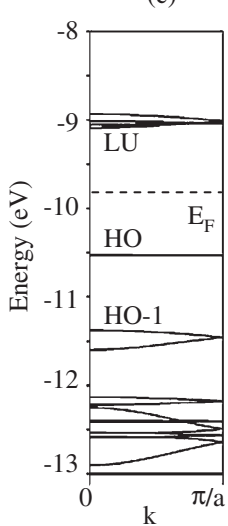

Figure 4. Band structures of (a) PFB, (b) PFPh, and (c) PFSu.

energy levels at $\mathrm{k}=\pi / a$ due to the asymmetry in a unit cell of the alternate copolymers. Furthermore, the energy level of the PO band formed by Co or Ni 3d orbitals decreases as compared with those of PFeC. On the other hand, the energy band formed by the $\pi$ orbitals of the $\mathrm{Cp}$ ring and $\mathrm{Fe} 3 \mathrm{~d}$ orbitals is slightly affected by the change in the transition metals. Their localized PO bands are energy levels caused by doped impurities in semiconductors. The band structures of metallocene-based

polymers including $\mathrm{Ru}$ or Os exhibited the same tendency as ferrocene-based polymers. As a result, the band structures of the alternate copolymers possess two superpositioned metallocene-based polymers. We have also calculated the band structures of ferrocene-based polymers that had bridging parts between the nearest $\mathrm{Cp}$ rings. The polymer with boron bridges is considered to possess similar electronic properties to PFS. On the other hand, the polymers with phosphorus or sulfur bridges possessed a flat $2 \mathrm{p}$ band whose COs were localized at the bridging parts. These localized bands, which have a low ionization potential, are considered to cause a high reactivity in the polymers.

Acknowledgment. The authors would like to thank Prof. R. Hoffmann and his coworkers for their permission to use the YAeHMOP program.

Received: August 13, 2008 Accepted: September 6, 2008 Published: October 22, 2008

\section{REFERENCES}

1. S. Barlow and D. O’Hare, Chem. Rev., 97, 637 (1997).

2. I. Manners, "Synthetic Metal-Containing Polymers," Wiley-VCH, Weinheim, Germany, 2004. 
3. T. Hirao, M. Kurashina, K. Aramaki, and H. Nishihara, J. Chem. Soc., Dalton Trans., 2929 (1996).

4. M. C. Böhm, J. Chem. Phys., 80, 2704 (1984).

5. Y. Matsuura and K. Matsukawa, Chem. Phys. Lett., 428, 321 (2006).

6. Y. Matsuura and K. Matsukawa, Chem. Phys. Lett., 447, 101 (2007).

7. R. Warratz, G. Peters, F. Studt, R. H. Römer, and F. Tuczek, Inorg. Chem., 45, 2531 (2006).

8. P. Jaitner, H. Schottenberger, S. Gamper, and D. Obendorf, J. Organomet. Chem., 475, 113 (1994).

9. Y. Matsuura and K. Matsukawa, Chem. Phys. Lett., 436, 224 (2007).

10. G. A. Landrum, "Yet Another extended Hückel Molecular Orbital Package (YAeHMOP)," Cornell University, 1997.

11. J. H. Ammeter, H.-B. Burgi, J. C. Thibeault, and R. Hoffmann, J. Am.
Chem. Soc., 100, 3686 (1978).

12. T. L. Cottrell, "The Strengths of Chemical Bonds," 2nd ed., Butterworths, London, 1958.

13. S. W. Benson, J. Chem. Educ., 42, 502 (1965).

14. Z.-F. Xu, Y. Xie, W.-L. Feng, and H. F. Schaefer, J. Phys. Chem., A, 107, 2716 (2003).

15. R. Hoffmann, C. Janiak, and C. Kollmar, Macromolecules, 24, 3725 (1991).

16. R. Warratz, G. Peters, F. Studt, R. H. Römer, and F. Tuczek, Inorg. Chem., 45, 2531 (2006).

17. P. A. Cox, "The Electrical Structure and Chemstry of Solids," Oxford University Press, Oxford, 1987. 